\title{
Adaptive Consensus Algorithm for Distributed Heat-Electricity Energy Management of an Islanded Microgrid
}

\author{
Xiaofeng Dong ${ }^{1,3}$, Xiaoshun Zhang ${ }^{2, *}$ and Tong Jiang ${ }^{1}$ \\ 1 Department of Electrical and Electronic Engineering, North China Electric Power University, \\ Beijing 100000, China; xiaofengdong1984@gmail.com (X.D.); jiangtong@ncepu.edu.cn (T.J.) \\ 2 College of Engineering, Shantou University, Shantou 515000, China \\ 3 State Grid Suzhou Power Supply Company, Suzhou 215004, China \\ * Correspondence: xszhang1990@gmail.com; Tel.: +86-150-1752-7246
}

Received: 10 July 2018; Accepted: 19 August 2018; Published: 26 August 2018

\begin{abstract}
This paper proposes a novel adaptive consensus algorithm (ACA) for distributed heat-electricity energy management (HEEM) of an islanded microgrid. In order to simultaneously satisfy the heat-electricity energy balance constraints, ACA is implemented with a switch between unified consensus and independent consensus according to the dynamic energy mismatches. The feasible operation region of a combined heat and power (CHP) unit is decomposed into eight searching sub-regions, thus its electricity and heat energy outputs can simultaneously match the incremental cost consensus requirement and the heat-electricity energy balance constraints. Case studies are thoroughly carried out to verify the performance of ACA for distributed HEEM of an islanded microgrid.
\end{abstract}

Keywords: adaptive consensus algorithm; distributed heat-electricity energy management; eight searching sub-regions; islanded microgrid

\section{Introduction}

Over the past decades, microgrids have attracted extensive attention and study as they provide an efficient and flexible way to integrate various distributed energy resources (DERs), local loads, and energy storage devices [1]. In general, a microgrid is a local energy grid which can be operated in either grid-connected or islanded modes [2]. When a microgrid is islanded, it needs to achieve an energy balance between the energy supply and the demand without the adequate power supply from the main grid [3].

In order to handle this issue, the economic dispatch (ED) is usually employed to minimize the total operating cost while satisfying various operating constraints (e.g., energy balance constraints) [4]. So far, ED of an islanded microgrid can be implemented with two frameworks, including the centralized and distributed frameworks. Under the first framework, the energy management system (EMS) needs to collect the operating parameters of all the energy suppliers and consumers [5], then an optimal dispatch scheme can be determined by a centralized optimization method. As a result, it will inevitably result in three critical problems:

- Communication bottleneck [6] due to the great increasing amount of data from the large integration of DERs;

- Expensive computation [7] for the growing controllable variables and operating constraints from the large integration of DERs;

- Low individual privacy and security [8]. 
Compared with the centralized ED, a distributed ED can automatically address all of the above problems [6]. Owing to this advantage, many distributed optimization techniques have been proposed for a distributed ED in a microgrid, such as distributed $\lambda$-iteration [9], population game method [10], and dual decomposition based optimization (DDO) [11]. Among these approaches, consensus-based algorithms were widely studied due to their remarkable self-organizing ability, significant robustness, and easy scalability [12-16]. In [12], a simple consensus-based optimization was designed for optimal resource management in an islanded microgrid. By considering the ramp rate limitations, a novel consensus and innovations [13] were presented for a multistep ED in a microgrid. Moreover, a novel consensus algorithm based ED was proposed by taking the impacts of communication time delays into account [15]. However, all of these consensus algorithms did not address two important issues:

- Multi-energy dispatch: The above ED only considers the electricity energy dispatch, and did not consider the optimal dispatch of other energies, e.g., the heat energy dispatch;

- Tight coupling features among various energies: As the participation of a combined heat and power (CHP) unit, the electricity and heat energy outputs are tightly coupled because of the feasible operation region constraint, which needs to be carefully designed in the distributed ED.

Therefore, this paper proposes a novel adaptive consensus algorithm (ACA) for distributed heat-electricity energy management (HEEM) of an islanded microgrid, which can not only realize the optimal multi-energy dispatch but also consider the tight coupling features between heat and electricity energies.

The remainder of this paper is organized as follows: Section 2 introduces the mathematical model of distributed HEEM, including the objective function, the operation constraints, and a detailed feature analysis of the incremental cost. Section 3 presents the optimization principle of ACA for distributed HEEM, while the detailed solving process is provided. Case studies on a microgrid with ten energy suppliers and seven energy consumers are given in Section 4, in which four optimization methods are introduced for performance comparison with ACA. Finally, Section 5 concludes the paper.

\section{Mathematical Model of Distributed HEEM}

In this study, the distributed HEEM aims to minimize the total operating cost of the entire islanded microgrid while satisfying the heat and electricity energy balance constraints and other operating constraints, as illustrated in Figure 1. Note that each controllable unit only communicates with the adjacent units during the computation of distributed HEEM, which is the main difference compared with the centralized ED [17].

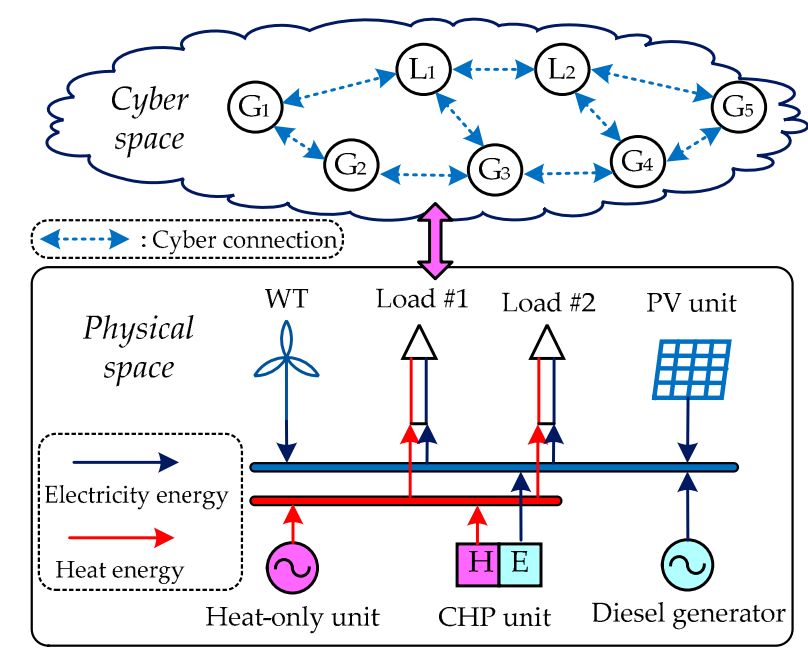

Figure 1. Framework of distributed heat-electricity energy management (HEEM) in an islanded microgrid. 


\subsection{Objective Function}

The total operating $\cos t f_{\text {total }}$ is equal to the sum of all the energy suppliers and consumers, which can be written as:

$$
f_{\text {total }}=\sum_{i \in \Omega_{\mathrm{G}}} f_{i}\left(P_{\mathrm{G} i}, H_{\mathrm{G} i}\right)+\sum_{i \in \Omega_{\mathrm{D}}} f_{i}\left(\Delta P_{\mathrm{D} i}\right),
$$

where $P_{\mathrm{G} i}$ and $H_{\mathrm{G} i}$ are the electricity and heat energy outputs of the $i$ th energy supplier, respectively; $\Delta P_{\mathrm{D} i}$ is electricity energy curtailment of the $i$ th energy consumer which participates in demand response (DR); $\Omega_{\mathrm{G}}$ and $\Omega_{\mathrm{D}}$ are the sets of the energy suppliers and consumers, respectively; and $f_{i}$ denotes the operating cost of the $i$ th energy supplier or consumer, which can be calculated as follows [18]:

$$
\begin{gathered}
f_{i}\left(P_{\mathrm{G} i}, H_{\mathrm{G} i}\right)= \begin{cases}0, & \text { for WT or PV unit } \\
\alpha_{i}+\beta_{i} P_{\mathrm{G} i}+\gamma_{i} P_{\mathrm{G} i}^{2} & \text { for diesel generator } \\
\alpha_{i}+\beta_{i} H_{\mathrm{G} i}+\gamma_{i} H_{\mathrm{G} i^{\prime}}^{2} & \text { for heat - only unit } \\
\alpha_{i}+\beta_{i} P_{\mathrm{G} i}+\gamma_{i} P_{\mathrm{G} i}^{2}+ & \\
\delta_{i} H_{\mathrm{G} i}+\theta_{i} H_{\mathrm{G} i}^{2}+\xi_{i} H_{\mathrm{G} i} P_{\mathrm{G} i}, & \text { for CHP unit }\end{cases} \\
f_{i}\left(\Delta P_{\mathrm{D} i}\right)=\frac{-1}{b_{i}} \Delta P_{\mathrm{D} i}^{2}+\frac{P_{\mathrm{D} i}^{0}-a_{i}}{b_{i}} \Delta P_{\mathrm{D} i}, i \in \Omega_{\mathrm{D}},
\end{gathered}
$$

where $\alpha_{i}, \beta_{i}, \gamma_{i}, \delta_{i}, \theta_{i}$, and $\xi_{i}$ are the operating cost coefficients of the $i$ th energy supplier; $a_{i}$ and $b_{i}$ are the operating cost coefficients of the ith energy consumer; WT and PV represent the the wind turbine and photovoltaic unit, respectively; and $P_{\mathrm{D} i}{ }^{0}$ is the current initial electricity energy demand of the $i$ th energy consumer.

\subsection{Constraints}

\subsubsection{Energy Balance Constraints}

The total energy outputs of all the energy supplier needs to match the total energy demands of all the energy consumers, is as follows:

$$
\begin{gathered}
\Delta E=\sum_{i \in \Omega_{\mathrm{G}}} P_{\mathrm{G} i}-\sum_{i \in \Omega_{\mathrm{D}}}\left(P_{\mathrm{D} i}^{0}-\Delta P_{\mathrm{D} i}\right)=0, \\
\Delta H=\sum_{i \in \Omega_{\mathrm{G}}} H_{\mathrm{G} i}-\sum_{i \in \Omega_{\mathrm{D}}} H_{\mathrm{D} i}=0,
\end{gathered}
$$

where $H_{\mathrm{D} i}$ is the heat energy demand of the $i$ th energy consumer; $\Delta E$ and $\Delta H$ are the electricity energy mismatch and heat energy mismatch, respectively, which will be combined into ACA in the latter section.

\subsubsection{Lower and Upper Capability Limits}

The energy outputs of each energy supplier, and the electricity energy curtailment of each energy consumer should be limited within their lower and upper bounds, as [18,19]:

$$
\begin{cases}P_{\mathrm{G} i}^{\min } \leq P_{\mathrm{G} i} \leq P_{\mathrm{G} i}^{\max }, & \text { for diesel generator } \\ H_{\mathrm{Gi}}^{\min } \leq H_{\mathrm{G} i} \leq H_{\mathrm{G} i}^{\max }, & \text { for heat }- \text { only unit } \\ P_{\mathrm{G} i}^{\min }\left(H_{\mathrm{G} i}\right) \leq P_{\mathrm{G} i} \leq P_{\mathrm{G} i}^{\max }\left(H_{\mathrm{G} i}\right), & \text { for CHP unit } \\ H_{\mathrm{G} i}^{\min }\left(P_{\mathrm{G} i}\right) \leq H_{\mathrm{G} i} \leq H_{\mathrm{G} i}^{\max }\left(P_{\mathrm{G} i}\right), & \text { for CHP unit } \\ \qquad 0 \leq \Delta P_{\mathrm{D} i} \leq \eta_{i} P_{\mathrm{D} i}^{0}, i \in \Omega_{\mathrm{D}},\end{cases}
$$

where $P_{\mathrm{D} i}{ }^{\min }$ and $P_{\mathrm{D} i}{ }^{\max }$ are the minimum and maximum electricity energy outputs of the $i$ th energy supplier, respectively; $H_{\mathrm{D} i}{ }^{\mathrm{min}}$ and $H_{\mathrm{D} i}{ }^{\mathrm{max}}$ are the minimum and maximum heat energy outputs of the 
$i$ th energy supplier, respectively; and $\eta_{i}$ is the maximum allowable electricity energy curtailment factor of the $i$ th energy consumer.

Note that both the WT and PV units are operated at their maximum power points under the current weather conditions [18], thus they do not require a consensus interaction with other controllable devices. Besides, it can be found from Equation (6) that both the lower and upper limits of the electrical energy output of CHP units are determined by different heat energy outputs and vice versa, which indicates that the energy outputs of $\mathrm{CHP}$ units should be enclosed by the boundary curve ABCD (i.e., the feasible operating region) [19], as shown in Figure 2.

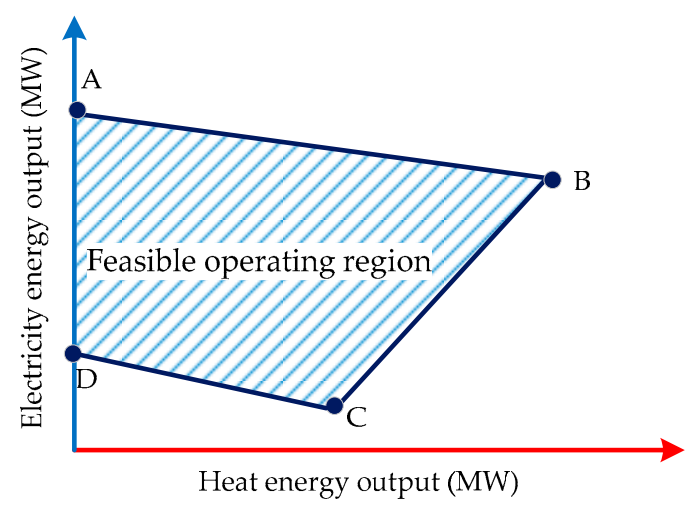

Figure 2. Feasible operating region of a combined heat and power (CHP) unit.

\subsection{Feature Analysis}

Since only the strictly convex feasible operating region is considered for each CHP unit, the proposed distributed HEEM is a strictly convex optimization with a unique optimum according to the quadratic objective functions Equations (1)-(3) and the linear constraints Equations (4)-(7). Hence, a feasible solution which simultaneously satisfies all the constraints can be regarded as the global optimum of distributed HEEM if all the energy suppliers and consumers can reach a consensus on the incremental cost, as [20]:

$$
\frac{\partial f_{1}\left(P_{\mathrm{G} 1}\right)}{\partial P_{\mathrm{G} 1}}=\cdots=\frac{\partial f_{i}\left(P_{\mathrm{G} i}\right)}{\partial P_{\mathrm{G} i}}=\frac{\partial f_{i}\left(H_{\mathrm{G} i}\right)}{\partial H_{\mathrm{G} i}}=\cdots=\frac{\partial f_{n}\left(\Delta P_{\mathrm{D} n}\right)}{\partial \Delta P_{\mathrm{D} n}}=\lambda,
$$

where $n$ is the number of controllable devices; and $\lambda$ is the incremental cost.

Note that such a consensus condition Equation (8) will not hold for a constrained optimization problem, as well as for distributed HEEM. In order to approximate the global optimum, all the constraints Equations (4)-(7) must be satisfied while the consensus condition should be satisfied as much as possible [20].

According to Equation (8), the incremental cost of each agent can be calculated as follows:

$$
\begin{gathered}
\left\{\begin{array}{c}
\lambda_{i}^{\mathrm{E}}=2 \gamma_{i} P_{\mathrm{G} i}+\beta_{i}, \quad \text { for diesel generator } \\
\lambda_{i}^{\mathrm{H}}=2 \gamma_{i} H_{\mathrm{G} i}+\beta_{i}, \quad \text { for heat }- \text { only unit }
\end{array},\right. \\
\left\{\begin{array}{l}
\lambda_{i}^{\mathrm{E}}=2 \gamma_{i} P_{\mathrm{G} i}+\xi_{i} H_{\mathrm{G} i}+\beta_{i}, \\
\lambda_{i}^{\mathrm{H}}=2 \theta_{i} H_{\mathrm{G} i}+\xi_{i} P_{\mathrm{G} i}+\delta_{i},
\end{array}\right. \text { for CHP unit, } \\
\lambda_{i}^{\mathrm{E}}=\frac{-2}{b_{i}} \Delta P_{\mathrm{D} i}+\frac{P_{\mathrm{D} i}^{0}-a_{i}}{b_{i}}, i \in \Omega_{\mathrm{D},}
\end{gathered}
$$

where $\lambda_{i}{ }^{\mathrm{E}}$ and $\lambda_{i}{ }^{\mathrm{H}}$ denote the electricity and heat incremental costs of the $i$ th energy supplier or consumer, respectively. 
Since all the operating cost coefficients except $b_{i}$ are the positive constants during each dispatch time interval, the incremental costs of the diesel generator, heat-only unit, and energy consumer, increase linearly with the electricity energy output, heat energy output, and electricity energy curtailment, respectively, as shown in Figure 3. In contrast, the electricity and heat incremental costs of the CHP unit are determined by both the electrical and heat energy outputs, as illustrated in Figure 4. For example, both the electrical and heat incremental costs will increase when the CHP unit is operated from the current operating point to the green region.

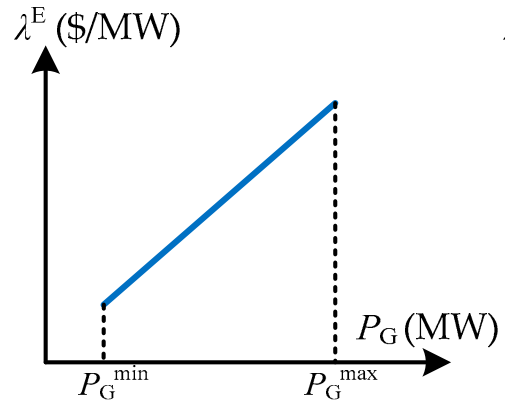

(a)

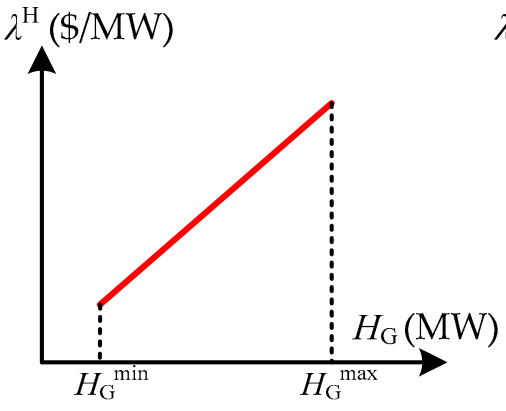

(b)

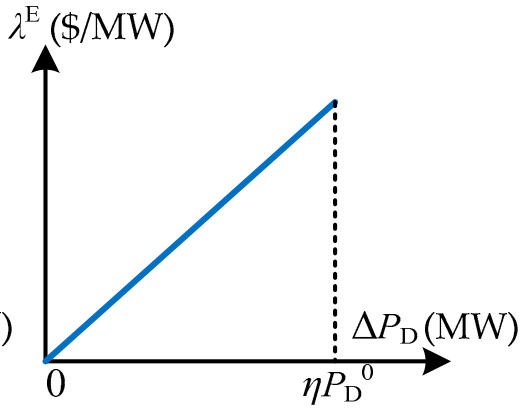

(c)

Figure 3. Incremental cost of various units. (a) Electrical incremental cost of diesel generator; (b) heat incremental cost of heat-only unit; (c) electrical incremental cost of energy consumer.

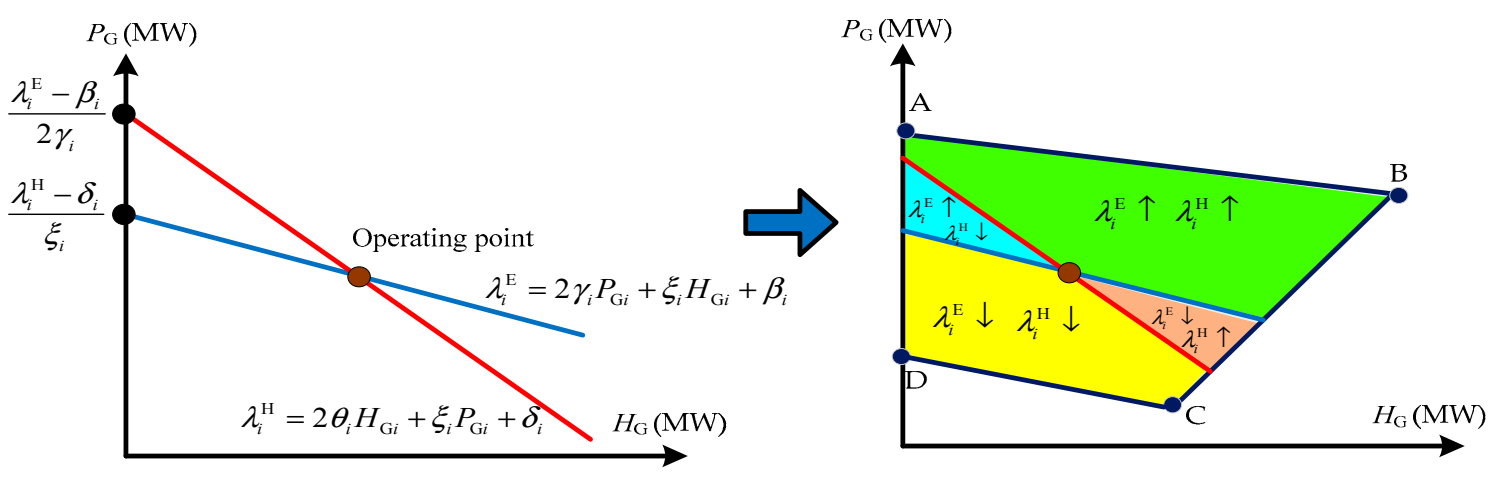

Figure 4. Illustration of incremental costs of a CHP.

\section{Design of ACA for Distributed HEEM}

\subsection{Graph Theory of Interaction Network}

The interaction network among different agents can be typically built with a directed graph $G=(V, E, A)$, where $V=\left\{v_{1}, v_{2}, \ldots, v_{n}\right\}$ is the set of nodes (agents); $E \subseteq V \times V$ denotes the edges (interactions); and $A=\left[a_{i j}\right] \in R^{n \times n}$ is a weighted adjacency matrix [21]. Based on these basic elements, the Laplacian matrix $L=\left[l_{i j}\right] \in R^{n \times n}$ and row stochastic matrix $D=\left[d_{i j}\right] \in R^{n \times n}$ of the graph $G$ can be determined as follows:

$$
\begin{gathered}
\left\{\begin{array}{l}
l_{i j}=-a_{i j}, \forall i \neq j \\
l_{i i}=\sum_{i=1, i \neq j}^{n} a_{i j},
\end{array}\right. \\
d_{i j}[k]=\left|l_{i j}\right| / \sum_{j=1}^{n}\left|l_{i j}\right|, i=1,2, \ldots, n
\end{gathered}
$$

where $k$ is the discrete time index. 
In this paper, the weighted adjacency matrix is set to be a simple $(0,1)$-matrix, thereby $a_{i j}=1$ if the $i$ th agent and the $j$ th agent communicate with each other, otherwise $a_{i j}=0$.

\subsection{Adaptive Consensus Algorithm}

The basic principle of ACA is that each agent aims to reach a consensus on a specific state with the adjacent agents by regulating its own state based on the current states from the adjacent agents. This process can be described by the first-order consensus, as [20]:

$$
x_{i}[k+1]=\sum_{j=1}^{n} d_{i j}[k] x_{j}[k]
$$

where $x_{i}$ is the state of the $i$ th agent, which refers to the incremental cost of each agent for distributed HEEM on the basis of Equation (8).

In this study, each agent will transmit its own energy output or demand to the microgrid EMS at each iteration, then EMS will update $\Delta E$ and $\Delta H$, and send them to each agent. In order to satisfy the energy balance constraints Equations (4) and (5), these two mismatches need to be fully considered in the consensus interaction among the agents, which can be achieved as follows:

- Unified consensus: If the signs of $\Delta E$ and $\Delta H$ are consistent, i.e., $\Delta E \Delta H \geq 0$, then all the agents can update their incremental cost state in a unified interaction network, as

$$
\lambda_{i}[k+1]=\left\{\begin{array}{ll}
\sum_{j=1}^{n} d_{i j}[k] \lambda_{j}[k]-\mu \Delta E, & i \in \Omega_{\mathrm{E}} \\
\sum_{j=1}^{n} d_{i j}[k] \lambda_{j}[k]-\mu \Delta H, & i \in \Omega_{\mathrm{H}}
\end{array},\right.
$$

- Independent consensus: If the signs of $\Delta E$ and $\Delta H$ are inconsistent, i.e., $\Delta E \cdot \Delta H<0$, then the electricity agents and heat agents need to be separated to update their incremental cost state in two independent interaction networks, as:

$$
\left\{\begin{array}{ll}
\lambda_{i}^{\mathrm{E}}[k+1]=\sum_{j \in \Omega_{\mathrm{E}}} d_{i j}^{\mathrm{E}}[k] \lambda_{j}^{\mathrm{E}}[k]-\mu \Delta E, & i \in \Omega_{\mathrm{E}} \\
\lambda_{i}^{\mathrm{H}}[k+1]=\sum_{j \in \Omega_{\mathrm{H}}} d_{i j}^{\mathrm{H}}[k] \lambda_{j}^{\mathrm{H}}[k]-\mu \Delta H, & i \in \Omega_{\mathrm{H}}
\end{array},\right.
$$

where $\Omega_{\mathrm{E}}$ and $\Omega_{\mathrm{H}}$ represent the sets of electricity agents and heat agents, respectively; $d_{i j}{ }^{\mathrm{E}}$ is the $(i, j)$ entry of the row stochastic matrix of the interaction network among the electricity agents; $d_{i j} \mathrm{H}$ is the $(i, j)$ entry of the row stochastic matrix of the interaction network among the heat agents; and $\mu$ denotes the adjustment factor of energy mismatch, $\mu>0$.

Therefore, each agent will regulate its incremental cost between these two consensus modes according to the sign of $(\Delta E \cdot \Delta H)$, as illustrated in Figure 5. After a series of consensus interactions by Equations (15) and (16), the energy balance constraints Equations (4) and (5) can be satisfied since both the electricity energy mismatch $\Delta E$ and heat energy mismatch $\Delta H$ will be sufficiently small. It is important that each interaction network should be strongly connected, i.e., any vertex can be realized from any other vertex by a directed path, thereby the consensus convergence can be guaranteed. 


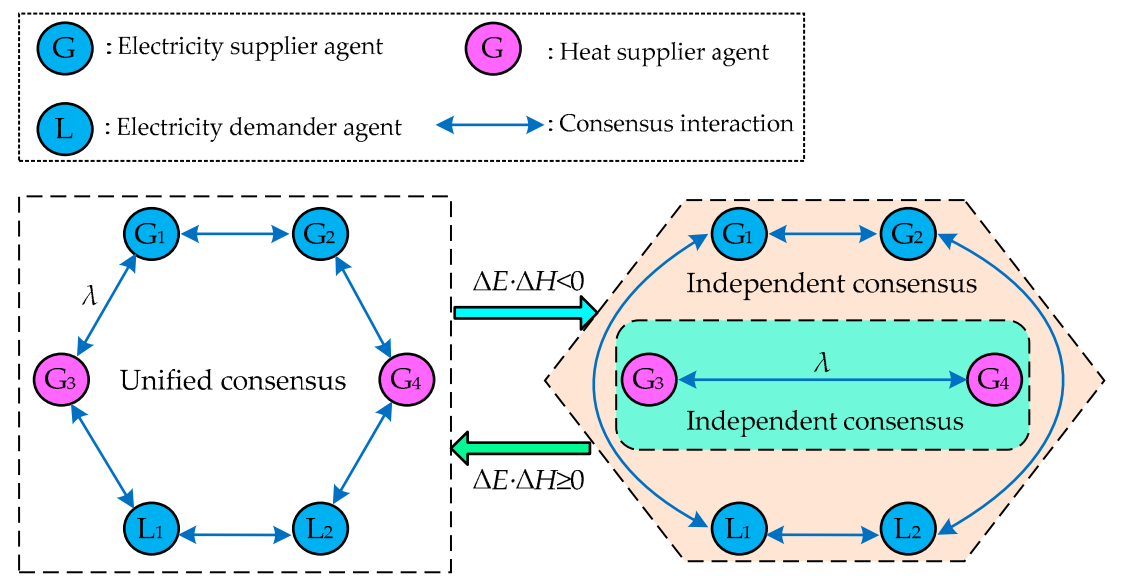

Figure 5. Principle of adaptive consensus algorithm.

\subsection{Constraints Handling}

Owing to the lower and upper capability limits Equations (6) and (7), all the agents may not reach a consensus on the incremental cost. Hence, a virtual incremental cost [17] is designed in ACA, which corresponds to the actual incremental cost of each agent. Note that each agent is responsible for computing its own incremental cost. More specifically, each agent can update its virtual incremental cost via a consensus interaction with adjacent agents by Equations (15) and (16), which is not limited by the constraints Equations (6) and (7). After updating the virtual incremental cost at each iteration, each agent can calculate its controllable variable by fully considering the constraints, while the actual incremental cost can be determined by Equations (9)-(11). Hence, all the constraints of distributed HEEM can be satisfied, while all the agents can reach a consensus on the incremental cost as much as possible.

1. Diesel generator: The electrical energy output can be modified as follows:

$$
\begin{gathered}
P_{\mathrm{G} i}^{\mathrm{c}}=\left(\lambda_{i}^{\mathrm{E}}-\beta_{i}\right) / 2 \gamma_{i}, \\
P_{\mathrm{G} i}=\left\{\begin{array}{l}
P_{\mathrm{G} i}^{\min }, \text { if } P_{\mathrm{G} i}^{\mathrm{c}}<P_{\mathrm{G} i}^{\min } \\
P_{\mathrm{G} i}^{\mathrm{c}} \text { if } P_{\mathrm{G} i}^{\min } \leq P_{\mathrm{G} i}^{\mathrm{c}} \leq P_{\mathrm{G} i}^{\max }, \\
P_{\mathrm{G} i}^{\max }, \text { if } P_{\mathrm{G} i}^{\mathrm{c}}>P_{\mathrm{G} i}^{\max }
\end{array}\right.
\end{gathered}
$$

where $P_{\mathrm{G} i}{ }^{\mathrm{c}}$ is the consensus value of the electrical energy output of the $i$ th energy supplier.

2. Heat-only unit: The heat energy output can be modified as follows:

$$
\begin{gathered}
H_{\mathrm{G} i}^{\mathrm{c}}=\left(\lambda_{i}^{\mathrm{H}}-\beta_{i}\right) / 2 \gamma_{i} \\
H_{\mathrm{G} i}= \begin{cases}H_{\mathrm{G} i}^{\min }, & \text { if } H_{\mathrm{G} i}^{\mathrm{c}}<H_{\mathrm{G} i}^{\min } \\
H_{\mathrm{G} i}^{\mathrm{c}}, & \text { if } H_{\mathrm{G} i}^{\min } \leq H_{\mathrm{G} i}^{\mathrm{c}} \leq H_{\mathrm{G} i}^{\max } \\
H_{\mathrm{G} i}^{\mathrm{max}}, & \text { if } H_{\mathrm{G} i}^{\mathrm{c}}>H_{\mathrm{G} i}^{\max }\end{cases}
\end{gathered}
$$

where $H_{\mathrm{G} i}{ }^{\mathrm{c}}$ is the consensus value of the heat energy output of the $i$ th energy supplier.

3. Energy consumer: The electricity energy curtailment can be modified as follows:

$$
\Delta P_{\mathrm{D} i}^{\mathrm{c}}=\left(P_{\mathrm{D} i}^{0}-a_{i}-b_{i} \lambda_{i}^{\mathrm{E}}\right) / 2,
$$




$$
\Delta P_{\mathrm{D} i}= \begin{cases}0, & \text { if } \Delta P_{\mathrm{D} i}^{\mathrm{c}}<0 \\ \Delta P_{\mathrm{D} i}^{\mathrm{c}}, & \text { if } 0 \leq \Delta P_{\mathrm{D} i}^{\mathrm{c}} \leq \eta_{i} P_{\mathrm{D} i}^{0} \\ \eta_{i} P_{\mathrm{D} i}^{0}, & \text { if } \Delta P_{\mathrm{D} i}^{\mathrm{c}}>\eta_{i} P_{\mathrm{D} i}^{0}\end{cases}
$$

where $\Delta P_{\mathrm{D} i}{ }^{\mathrm{C}}$ is the consensus value of the electrical energy curtailment of the $i$ th energy consumer.

4. CHP unit: Since the electrical and heat energy outputs are highly coupled, the incremental cost should be controlled to meet the energy balance constraints and the feasible operating region constraint. Hence, the feasible operating region is decomposed into eight searching sub-regions, See Figure 6, allowing the CHP unit to adjust its energy outputs based on the current energy mismatches and the consensus value of incremental costs, as given in Table 1.

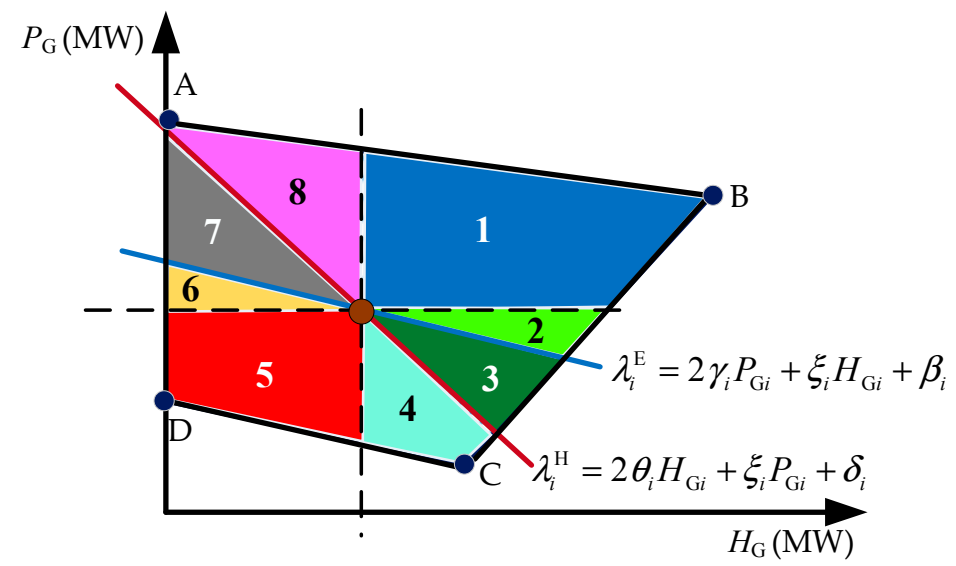

Figure 6. Eight searching sub-regions of the CHP unit.

Table 1. Adjusting rules of energy outputs of CHP unit.

\begin{tabular}{ccccc}
\hline $\boldsymbol{\Delta E} \boldsymbol{\mathbf { 0 }}$ & $\boldsymbol{\Delta H}>\mathbf{0}$ & $\lambda_{\boldsymbol{i}}{ }^{\mathrm{E}}[k]>\lambda_{\boldsymbol{i}}^{\mathrm{AE}}[k-\mathbf{1}]$ & $\lambda_{\boldsymbol{i}}{ }^{\mathrm{H}}[k]>\lambda_{i}{ }^{\mathrm{AH}}[k-\mathbf{1}]$ & $\left(\boldsymbol{P}_{\mathrm{Gi}}, \boldsymbol{H}_{\mathrm{G} i}\right)$ \\
\hline True & True & True & True & No adjustment \\
True & True & True & False & No adjustment \\
True & True & False & True & No adjustment \\
True & True & False & False & Sub-region \#5 \\
True & False & True & True & Sub-region \#2 \\
True & False & True & False & No adjustment \\
True & False & False & True & Sub-region \#3 \\
True & False & False & False & Sub-region \#4 \\
False & True & True & True & Sub-region \#8 \\
False & True & True & False & Sub-region \#7 \\
False & True & False & True & No adjustment \\
False & True & False & False & Sub-region \#6 \\
False & False & True & True & Sub-region \#1 \\
False & False & True & False & No adjustment \\
False & False & False & True & No adjustment \\
False & False & False & False & No adjustment \\
\hline
\end{tabular}

Note that the CHP unit does not need to adjust its electrical and heat energy outputs if the consensus requirement and energy balance constraints cannot be satisfied simultaneously. For instance, when both the current virtual incremental costs $\left(\lambda_{i}{ }^{\mathrm{E}}[k], \lambda_{i}{ }^{\mathrm{H}}[k]\right)$ are larger than the last actual incremental $\operatorname{costs}\left(\lambda_{i}{ }^{\mathrm{AE}}[k-1], \lambda_{i}{ }^{\mathrm{AH}}[k-1]\right)$, while both the energy mismatches are positive $(\Delta E>0, \Delta H>0)$, then the $\mathrm{CHP}$ unit will readjust the energy balances by increasing its incremental costs, thus its electrical and heat energy outputs will remain unchanged. In addition, when the CHP unit needs to adjust its energy outputs, the electrical and heat energy outputs can be updated according to the energy mismatches, as follows: 


$$
\left\{\begin{array}{l}
P_{\mathrm{G} i}[k+1]=P_{\mathrm{G} i}[k]-\mu_{\mathrm{E}} \Delta E \\
H_{\mathrm{G} i}[k+1]=H_{\mathrm{G} i}[k]-\mu_{\mathrm{H}} \Delta H
\end{array},\right.
$$

where $\mu_{\mathrm{E}}$ and $\mu_{\mathrm{H}}$ denote the adjustment factors of electrical and heat energy outputs, respectively.

If the operating point of the CHP unit is beyond the corresponding sub-region, then the electrical and heat energy outputs should be modified by the closest point (the point with the shortest Euclidean distance to the updated operating point) within the sub-region.

\subsection{Execution Procedure}

To sum up, the detailed execution procedure of ACA for distributed HEEM of an islanded microgrid is given in Algorithm 1, where $\tau$ is the energy mismatch tolerance, which is set to be 0.001 in this paper.

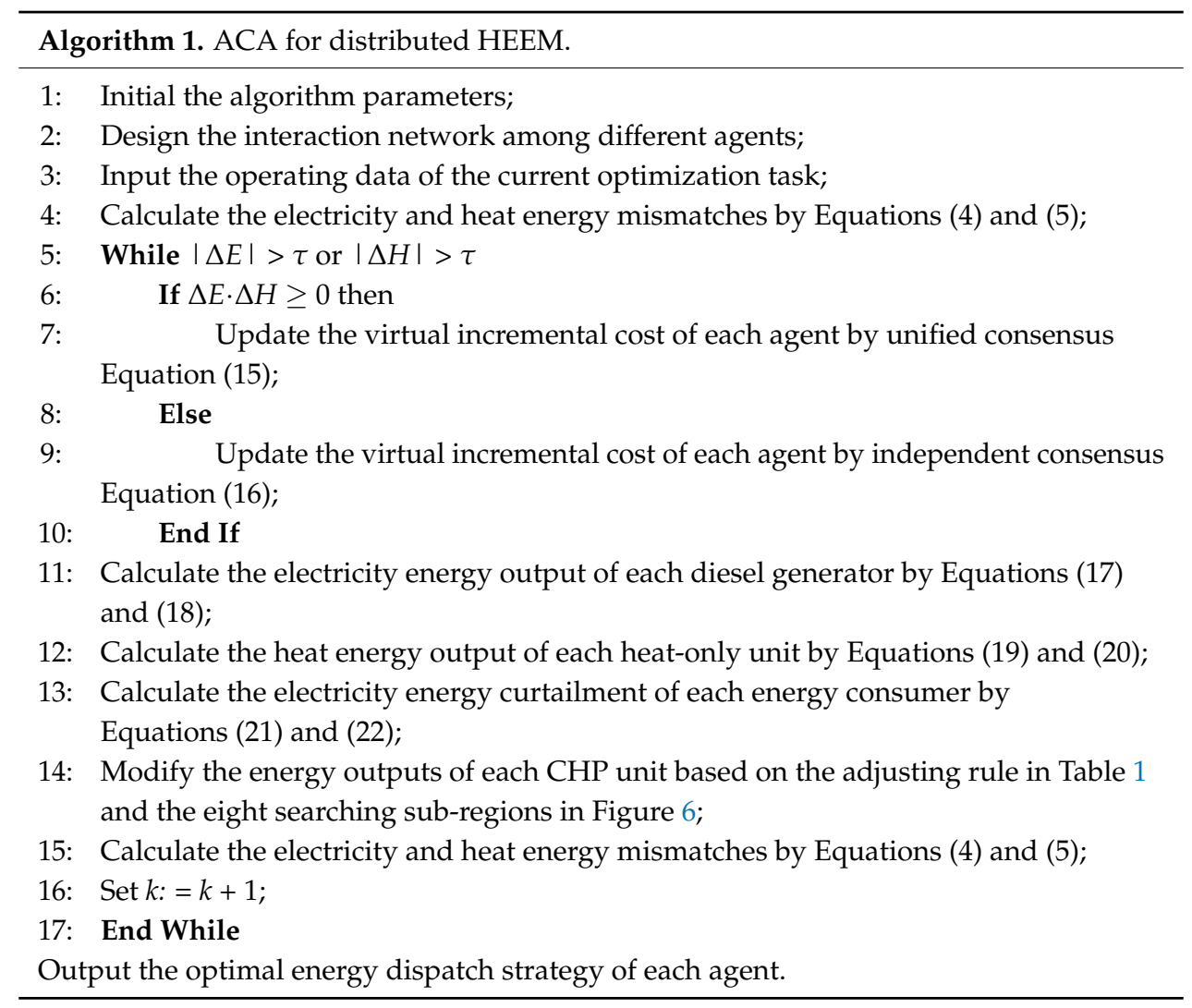

\section{Case Studies}

\subsection{Simulation Model}

In order to test the multi-energy dispatch, the islanded microgrid [18] with three PV units, two WTs, two diesel generators, one heat-only unit, two CHP units, and seven controllable energy consumers, is used for the simulation. Hence, both the electrical and heat parts are simultaneously considered in simulation, where the detailed mathematical model of distributed HEEM can be constructed by acquiring the operating constraints and operating cost function of energy for each supplier or consumer. Furthermore, the operating cost coefficients are given in Table 2; the physical topology is provided in Figure 7, and the interaction network among them is illustrated in Figure 8. In addition, three operating scenarios (i.e., scenarios \#1 to \#3) with different energy outputs of renewables (i.e., $0.8,0.6$, and $1 \mathrm{MW}$ ), instead of a single operating scenario, are designed for evaluating the optimization performance of different algorithms more scientifically. The adjustment factors $\mu$, $\mu_{\mathrm{E}}$, and $\mu_{\mathrm{H}}$ are set to be $10,0.1$, and 0.1 , respectively. The following simulations will be carried out in 
Matlab R2016a by a personal computer with Intel(R) Xeon (R) E5-2670 v3 CPU at $2.3 \mathrm{GHz}$ with 64 GB of RAM.

Table 2. Operating cost coefficients of controllable units.

\begin{tabular}{cccccccc}
\hline Type & No. & $\alpha_{i}$ & $\beta_{i}$ & $\gamma_{i}$ & $\delta_{i}$ & $\boldsymbol{\theta}_{\boldsymbol{i}}$ & $\zeta_{\boldsymbol{i}}$ \\
\hline \multirow{2}{*}{ Diesel generator } & $\mathrm{G}_{1}$ & 10.193 & 210.36 & 250.2 & - & - & - \\
& $\mathrm{G}_{2}$ & 2.305 & 301.4 & 1100 & - & - & - \\
\hline \multirow{2}{*}{ Heat-only unit } & $\mathrm{G}_{3}$ & 33 & 12.3 & 6.9 & - & - & - \\
\hline \multirow{2}{*}{ CHP unit } & $\mathrm{G}_{4}$ & 339.5 & 185.7 & 44.2 & 53.8 & 38.4 & 40 \\
& $\mathrm{G}_{5}$ & 100 & 288 & 34.5 & 21.6 & 21.6 & 8.8 \\
\hline \multirow{5}{*}{ Energy } & $\mathrm{L}_{1}$ & 1 & -0.002 & - & - & - & - \\
consumer & $\mathrm{L}_{2}$ & 1 & -0.002 & - & - & - & - \\
& $\mathrm{L}_{3}$ & 1 & -0.001 & - & - & - & - \\
& $\mathrm{L}_{4}$ & 1 & -0.001 & - & - & - & - \\
& $\mathrm{L}_{5}$ & 1 & -0.001 & - & - & - & - \\
& $\mathrm{L}_{6}$ & 1 & -0.0035 & - & - & - & - \\
& $\mathrm{L}_{7}$ & 1 & -0.0035 & - & - & - & - \\
\hline
\end{tabular}

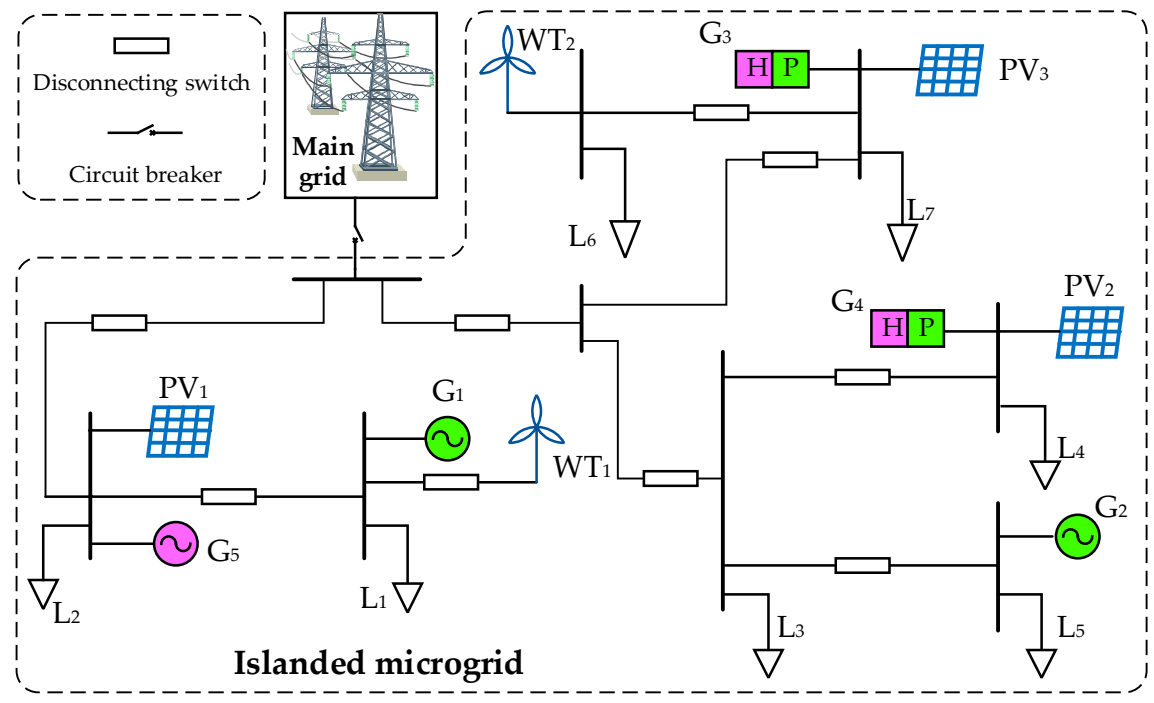

Figure 7. Physical topology of the testing islanded microgrid.

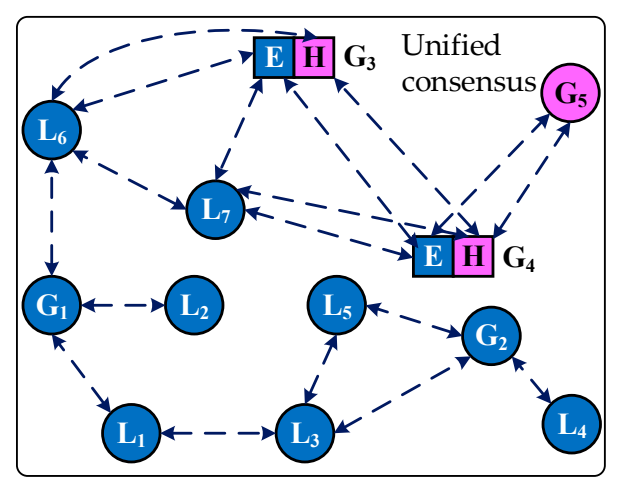

(a)

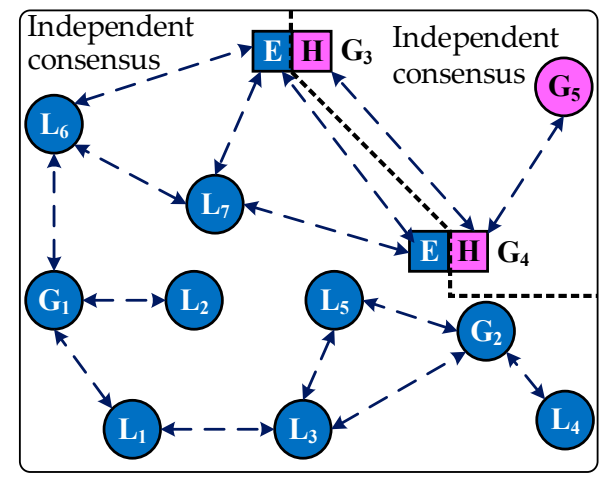

(b)

Figure 8. Interaction network of different agents. (a) Unified consensus; (b) independent consensus. 


\subsection{Study of Convergence}

This case study is executed to reveal the convergence of ACA. Figure 9 shows the convergence process of ACA for distributed HEEM under scenario \#1. It can be found from Figure 9a that the virtual incremental cost of each agent will update between unified consensus mode and independent consensus mode according to the dynamic energy mismatches, in which the incremental heat costs cannot reach a consensus with other incremental electrical costs due to the energy balance constraints Equations (4) and (5). Besides, some energy agents have reached their energy capability limits after a few interactions, as shown in Figure 9b. Moreover, two CHP units can adaptively adjust their energy outputs based on the adjusting rule in Table 1, see Figure 9c, where the zero searching sub-region indicates that the energy outputs of the CHP unit remain unchanged. Finally, both the electrical and heat energy mismatches $(\Delta E$ and $\Delta H)$ can simultaneously satisfy the energy mismatch tolerance after approximately 150 iterations, see Figure 9 d, i.e., $|\Delta E|<\tau$ and $|\Delta H|<\tau$. All of this proves that the convergence of ACA can be effectively guaranteed, while the consensus requirement and all the constraints Equations (4)-(7) can be fully satisfied.

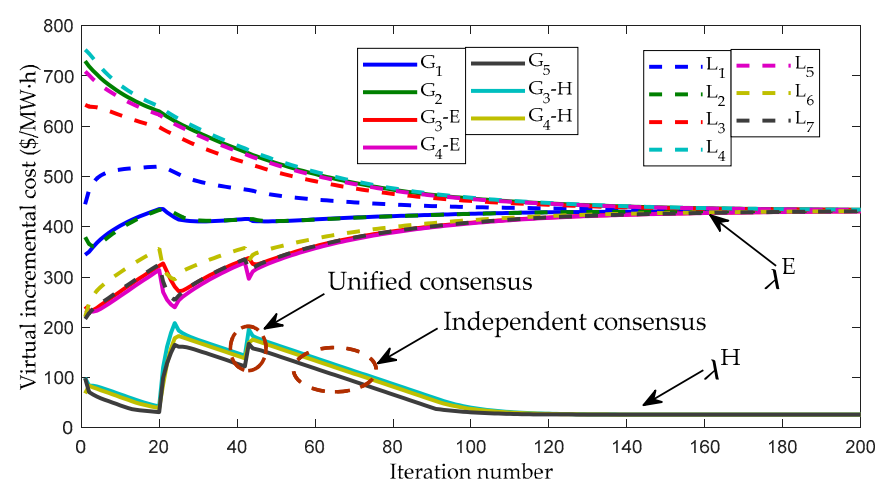

(a)

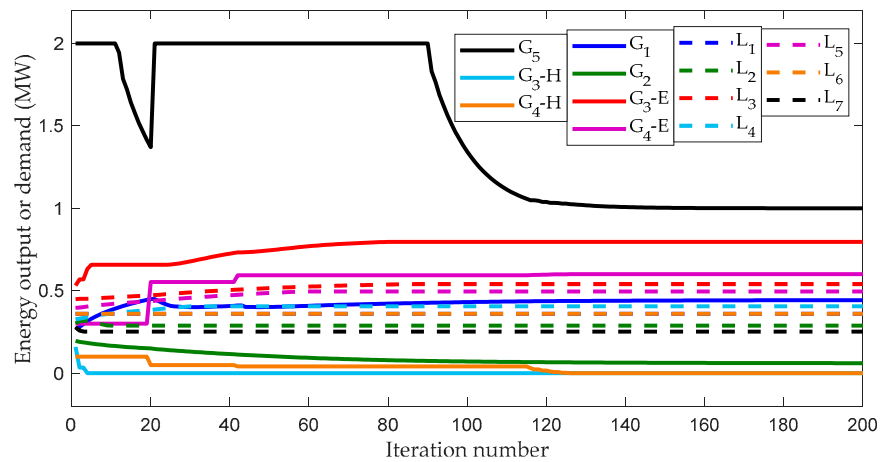

(b)

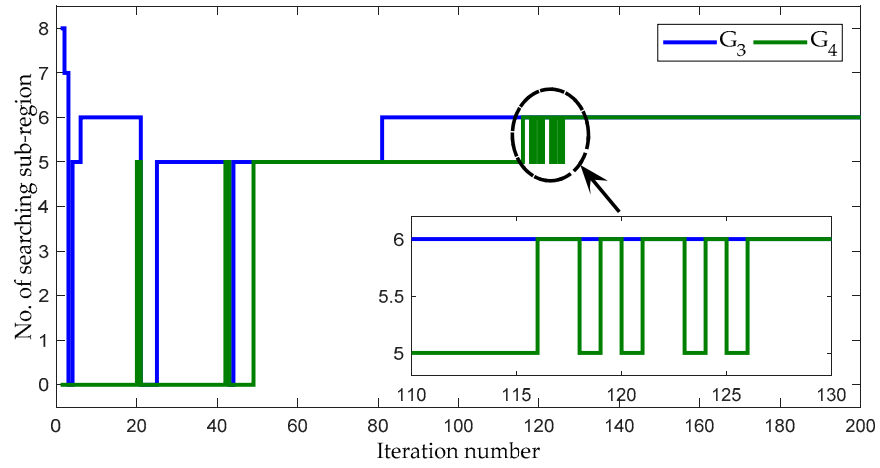

(c)

Figure 9. Cont. 


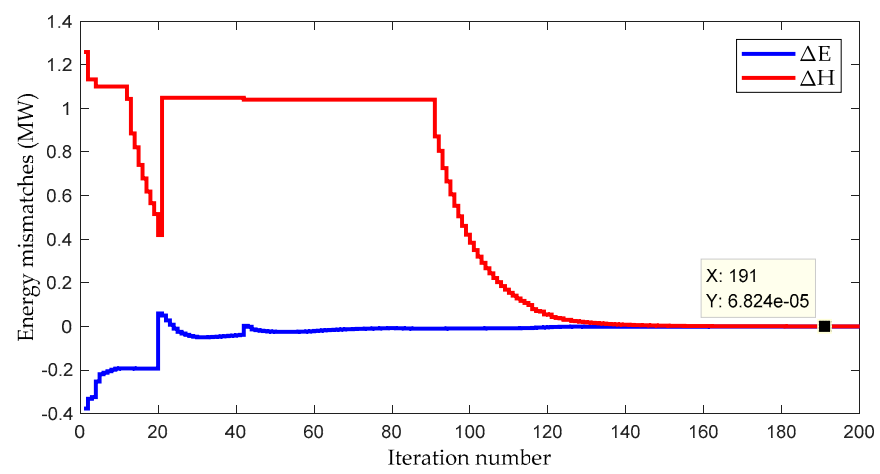

(d)

Figure 9. Convergence process of ACA for distributed HEEM. (a) Virtual incremental cost; (b) energy output or demand; (c) no. of searching sub-region of CHP units; (d) energy mismatches.

Here, the implementation period of distributed HEEM is set as $2 \mathrm{~s}$ for testing the real-time optimization performance of ACA. Note that the total time of each iteration includes the calculation time and information transmission time, which can be set as $1 \mathrm{~ms}$ with a conservative estimation. Figure 10 gives the real-time optimization of distributed HEEM obtained by ACA under three different scenarios as the total energy output of PV and WT units varies. It also verifies that ACA can converge to an optimal solution of distributed HEEM, while it can fully satisfy the real-time optimization of distributed HEEM because its convergence time is much shorter than the implementation period. Furthermore, it is clear that the incremental electrical cost decreases with the increasing electrical energy outputs of renewables.

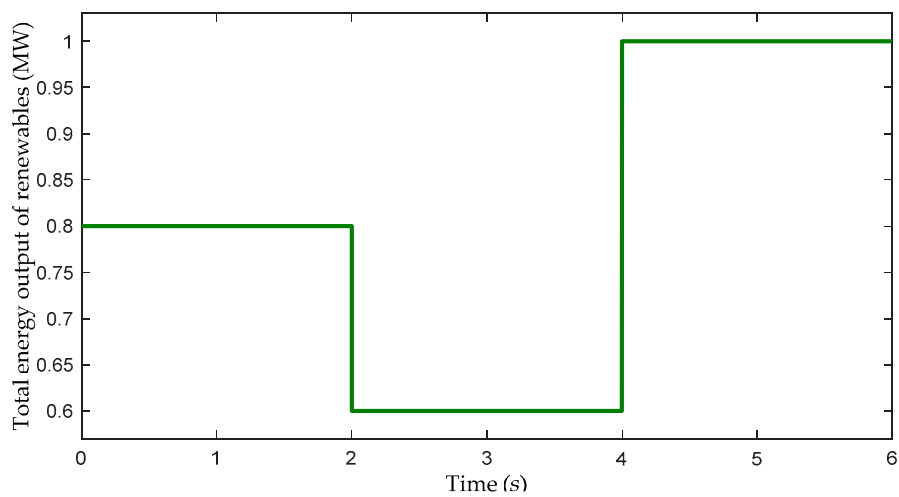

(a)

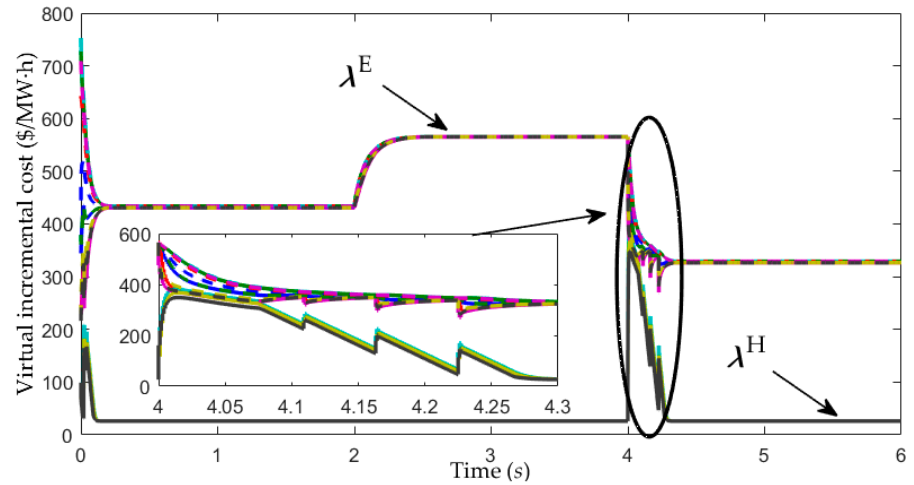

(b)

Figure 10. Real-time optimization of distributed HEEM by ACA. (a) Electrical energy fluctuation of renewables; (b) virtual incremental cost. 


\subsection{Comparative Results and Discussions}

In order to further test the performance of ACA, four optimization algorithms, including genetic algorithm (GA) [22], interior point method (IPM) [23], distributed particle swarm optimization (DPSO) [24], and DDO [11], are introduced for comparisons, where the first two methods are centralized; and the latter two methods are distributed.

Table 3 provides the dispatch strategies obtained by different algorithms under scenario \#1. It illustrates that ACA can converge to a high-quality optimum of distributed HEEM, which is very similar to the global optimum obtained by centralized IPM. Furthermore, the quality of the obtained optimum of GA is the lowest due to its premature convergence. This also demonstrates the effectiveness of ACA for distributed HEEM.

Table 3. Obtained dispatch strategies under scenario \#1.

\begin{tabular}{ccccccc}
\hline \multirow{2}{*}{ No. } & Energy Type & \multicolumn{5}{c}{ Dispatch Strategy (MW) } \\
\cline { 3 - 7 } & & GA & IPM & DPSO & DDO & ACA \\
\hline $\mathrm{G}_{1}$ & Electrical & 0.4013 & 0.3134 & 0.4150 & 0.3178 & 0.4427 \\
\hline $\mathrm{G}_{2}$ & Electrical & 0.1617 & 0.0492 & 0.2000 & 0.0500 & 0.0602 \\
\hline $\mathrm{G}_{3}$ & Electrical & 0.8448 & 0.9963 & 1.0000 & 0.9923 & 0.7962 \\
& Heat & 0.3182 & 0.0056 & 0.0000 & 0.0069 & 0.0000 \\
\hline $\mathrm{G}_{4}$ & Electrical & 0.4588 & 0.5946 & 0.6000 & 0.5941 & 0.5999 \\
& Heat & 0.2952 & 0.0326 & 0.0000 & 0.0354 & 0.0000 \\
\hline $\mathrm{G}_{5}$ & Heat & 0.3858 & 0.9619 & 1.0000 & 0.9577 & 1.0000 \\
\hline $\mathrm{L}_{1}$ & Electrical & 0.3938 & 0.3771 & 0.4500 & 0.3744 & 0.3600 \\
\hline $\mathrm{L}_{2}$ & Electrical & 0.3220 & 0.3310 & 0.3600 & 0.3353 & 0.2880 \\
\hline $\mathrm{L}_{3}$ & Electrical & 0.4736 & 0.5359 & 0.5400 & 0.5360 & 0.5400 \\
\hline $\mathrm{L}_{4}$ & Electrical & 0.3624 & 0.4030 & 0.4050 & 0.4034 & 0.4050 \\
\hline $\mathrm{L}_{5}$ & Electrical & 0.4395 & 0.4919 & 0.4950 & 0.4921 & 0.4950 \\
\hline $\mathrm{L}_{6}$ & Electrical & 0.3922 & 0.3614 & 0.4500 & 0.3605 & 0.3600 \\
\hline $\mathrm{L}_{7}$ & Electrical & 0.2840 & 0.2531 & 0.3150 & 0.2524 & 0.2520 \\
\hline$H_{\mathrm{D}}$ & Heat & 1 & 1 & 1 & 1 & 1 \\
\hline $\mathrm{PV}$ & Electrical & 0.3 & 0.3 & 0.3 & 0.3 & 0.3 \\
\hline $\mathrm{WT}$ & Electrical & 0.5 & 0.5 & 0.5 & 0.5 & 0.5 \\
\hline $\mathrm{Total}_{\mathrm{n}}$ operating cost $(\$ / \mathrm{h})$ & 1201.48 & 1091.38 & 1153.99 & 1091.57 & 1113.91 \\
\hline & & & & & &
\end{tabular}

Table 4 gives the comparison results obtained by three algorithms under different scenarios in 100 runs. It shows that IPM, DDO, and ACA always converge to the same optimum with a given initial solution and parameters as they are essentially the deterministic optimization algorithms. In contrast, both GA and DPSO often search different optimums in different runs due to their random heuristic operators. Furthermore, these two heuristic optimization algorithms also result in a much longer execution time than that of the other three methods, where the execution time of DPSO is shorter than that of GA due to its higher computation efficiency and distributed feature. Besides, the quality of optimum obtained by DDO is only lower than that of IPM, but its execution time is about four times that of ACA. Similarly, the optimum obtained by ACA is similar to the global optimum obtained by IPM, while the execution time is nearly the same. Hence, ACA is very suitable to yield the distributed HEEM because of its excellent performance regarding optimum quality and execution time. 
Table 4. Comparison results obtained by five algorithms under different scenarios in 100 runs.

\begin{tabular}{ccccccc}
\hline \multirow{2}{*}{ Scenario No. } & Algorithm & Type & Execution Time (s) & \multicolumn{2}{c}{ Total Operating Cost (\$/h) } \\
\cline { 5 - 7 } & & & & Max & Avg & Min \\
\hline \multirow{3}{*}{$\# 1$} & GA & Centr. & 9.45 & 1250.29 & 1207.94 & 1148.17 \\
& IPM & Centr. & 0.32 & 1091.38 & 1091.38 & 1091.38 \\
& DPSO & Distr. & 4.17 & 1153.99 & 1151.90 & 1124.50 \\
& DDO & Distr. & 1.27 & 1091.57 & 1091.57 & 1091.57 \\
& ACA & Distr. & 0.20 & 1113.91 & 1113.91 & 1113.91 \\
\hline \multirow{3}{*}{$\# 2$} & GA & Centr. & 9.16 & 1334.22 & 1299.12 & 1251.35 \\
& IPM & Centr. & 0.56 & 1168.46 & 1168.46 & 1168.46 \\
& DPSO & Distr. & 4.14 & 1228.44 & 1228.15 & 1226.12 \\
& DDO & Distr. & 1.49 & 1198.32 & 1198.32 & 1198.32 \\
$\# 3$ & ACA & Distr. & 0.51 & 1212.54 & 1212.54 & 1212.54 \\
\hline & GA & Centr. & 9.36 & 1178.54 & 1135.87 & 1083.35 \\
& IPM & Centr. & 0.26 & 1020.67 & 1020.67 & 1020.67 \\
& DPSO & Distr. & 4.42 & 1080.39 & 1078.45 & 1041.42 \\
& DDO & Distr. & 1.24 & 1020.77 & 1020.77 & 1020.77 \\
& ACA & Distr. & 0.34 & 1024.73 & 1024.73 & 1024.73 \\
\hline
\end{tabular}

\subsection{Scalability Test of ACA}

This case study is used for testing the scalability of ACA for a larger scale system. In general, ACA will lead to a slower convergence rate for a larger scale microgrid with more agents. For testing the scalability of ACA, different scales of microgrid are designed based on the presented microgrid with 12 agents, in which the scales are 5, 10,50, 100, and 500 times of the presented microgrid, respectively. Figure 11 shows the convergence process of ACA for two scales of microgrids under scenarios \#3. It can be found that ACA can also converge to the optimal virtual incremental costs when the number of agents increases from 12 to 600 . Although the number of agents increases by fifty-fold, the iteration number of convergence only increases from 340 to 488 . In addition, Figure 12 provides statistical results of iteration number of convergence under different numbers of agents by ACA. Similarly, it shows that the iteration number of convergence increases marginally as the number of agents increases from 12 to 6000 under different scenarios. More specifically, the iteration number of convergence with 6000 agents is only 2.8 times of that with 12 agents under scenario \#1. This reveals that ACA is suitable for real-world application with a high number of agents due to its superior scalability.

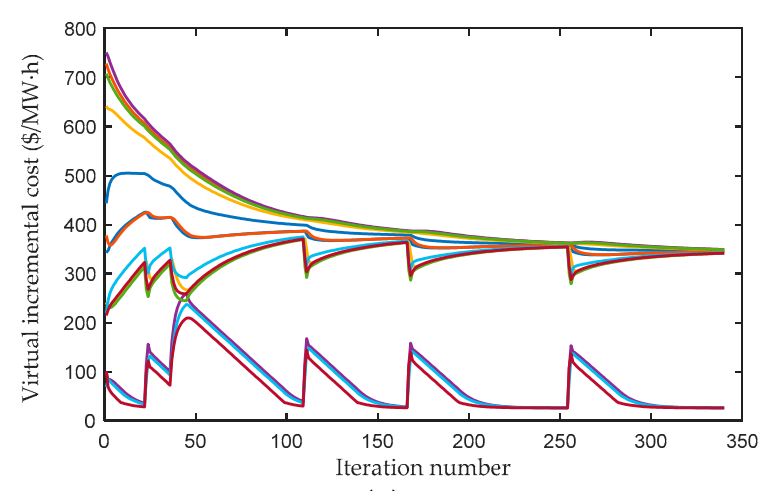

(a)

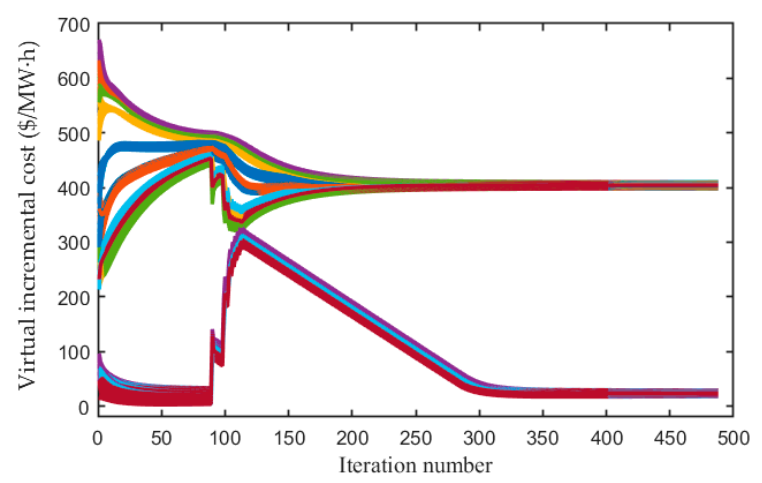

(b)

Figure 11. Convergence process of ACA under scenario \#3. (a) 12 agents; (b) 600 agents. 


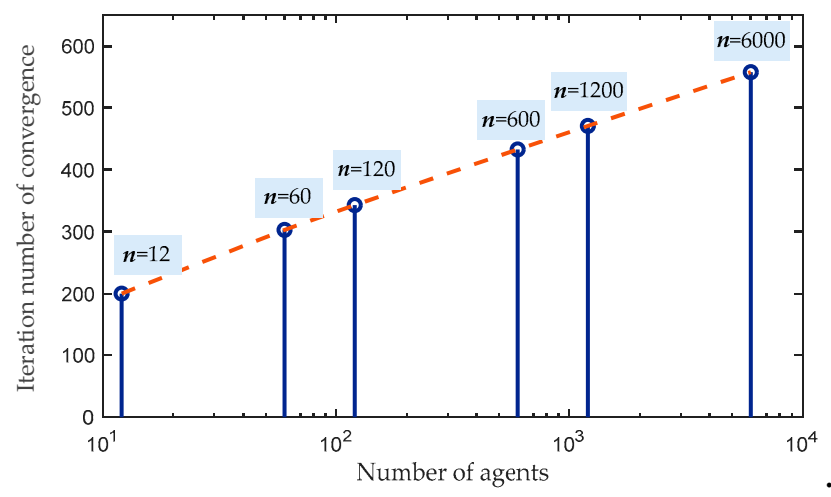

(a)

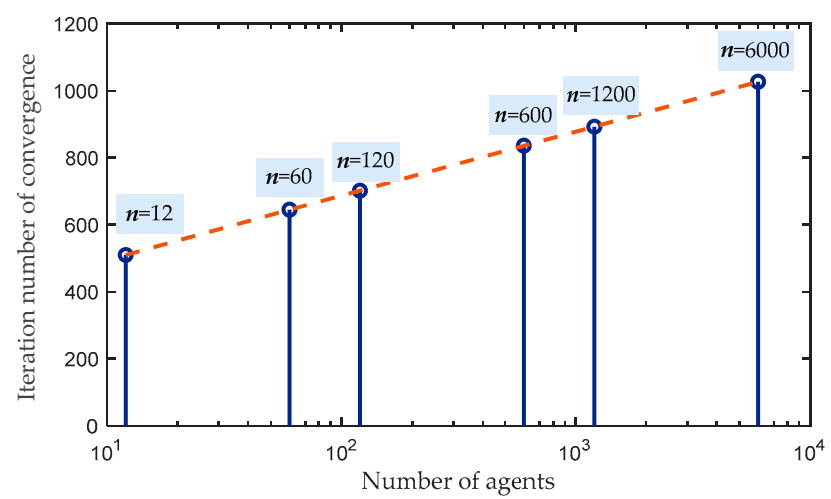

(b)

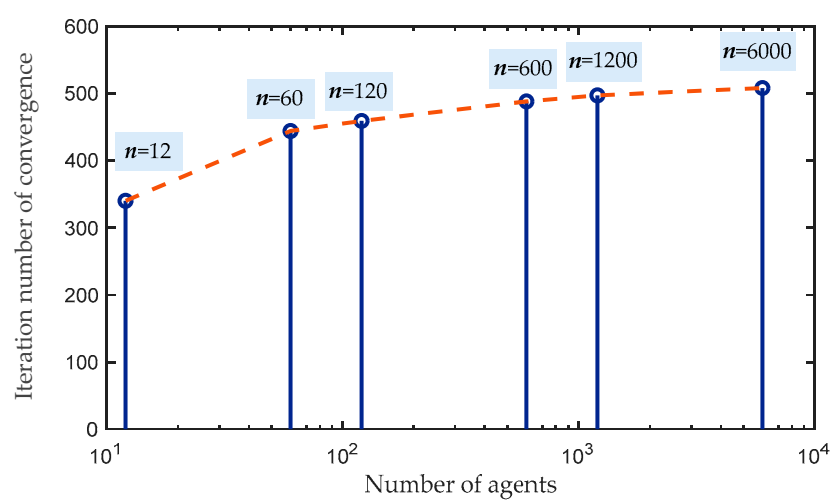

(c)

Figure 12. Iteration number of convergence under different numbers of agents by ACA. (a) Scenario \#1; (b) Scenario \#2; (c) Scenario \#3.

\section{Conclusions}

In summary, this paper presents a novel ACA for distributed HEEM of an islanded microgrid, which has the following contributions:

1. The ACA based distributed HEEM can effectively address the multi-energy dispatch of an islanded microgrid in a simple distributed manner, while various constraints (e.g., the tight coupling features among various energies) can be completely satisfied.

2. The proposed eight searching sub-regions effectively make the CHP unit adaptively adjust its energy outputs to simultaneously meet the consensus requirement and the heat-electricity energy balance constraints. 
3. Through the switch between unified consensus and independent consensus, ACA gradually converges to the optimal solution of the whole system according to the dynamic energy mismatches.

4. ACA can not only obtain a high-quality optimum of distributed HEEM, but also guarantee a short execution time. Hence, it can be generalized to be applied to other real-time distributed optimization issues of integrated energy systems.

Our future work will focus on improving the flexibility and generality of ACA by combining the model-free heuristic search or machine-learning mechanisms. Hence, it can handle more complex distributed optimization with high nonlinearity and nonconvexity, discontinuous and nondifferentiable objective functions.

Author Contributions: Data curation, X.D.; Investigation, X.Z.; Methodology, X.Z.; Project administration, X.D. and T.J.; Supervision, T.J.; Writing-original draft, X.Z.

Funding: This research was funded by [State Grid Corporation of China Science and Technology Project] grant number [2017-142].

Acknowledgments: The authors would like to gratefully acknowledge the State Grid Corporation of China.

Conflicts of Interest: The authors declare no potential conflict of interest.

\section{References}

1. Alsaidan, I.; Alannazi, A.; Gao, W.; Wu, H.; Khodaei, A. State-of-the-art in microgrid-integrated distributed energy storage sizing. Energies 2017, 10, 1421. [CrossRef]

2. Kim, H.-M.; Lim, Y.; Kinoshita, T. An intelligent multiagent system for autonomous microgrid operation. Energies 2012, 5, 3347-3362. [CrossRef]

3. Gao, H.; Chen, Y.; Xu, Y.; Liu, C.-C. Dynamic load shedding for islanded microgrid with limited generation resources. IET Renew. Power Gener. 2016, 10, 2953-2961. [CrossRef]

4. Lin, W.-M.; Tu, C.-S.; Tsai, M.-T. Energy management strategy for microgrid by using enhanced bee colony optimization. Energies 2016, 9, 5. [CrossRef]

5. Maghsoodlou, F.; Masiello, R.; Ray, T. Energy management systems. IEEE Power Energy Mag. 2004, 2, 49-57. [CrossRef]

6. Loia, V.; Vaccaro, A. Decentralized economic dispatch in smart grids by self-organizing dynamic agents. IEEE Trans. Syst. Man Cybern. Syst. 2014, 44, 397-408. [CrossRef]

7. Zheng, W.; Wu, W.; Zhang, B.; Sun, H.; Liu, Y. A fully distributed reactive power optimization and control method for active distribution networks. IEEE Trans. Smart Grid 2016, 7, 1021-1033. [CrossRef]

8. Amin, S.M. Smart grid security, privacy, and resilient architectures: Opportunities and challenges. In Proceedings of the 2012 IEEE Power and Energy Society General Meeting, San Diego, CA, USA, 22-26 July 2012; pp. 1-2.

9. Hu, J.; Chen, M.Z.Q.; Cao, J.; Guerrero, J.M. Coordinated active power dispatch for a microgrid via distributed lambda iteration. IEEE Trans. Emerg. Sel. Top. Circuits Syst. 2017, 7, 250-261. [CrossRef]

10. Mojica-Nava, E.; Barreto, C.; Quijano, N. Population games methods for distributed control of microgrids. IEEE Trans. Smart Grid 2015, 6, 2586-2595. [CrossRef]

11. Yang, Z.; Wu, R.; Yang, J.; Long, K.; You, P. Economical operation of microgrid with various devices via distributed optimization. IEEE Trans. Smart Grid 2016, 7, 857-867. [CrossRef]

12. $\mathrm{Xu}, \mathrm{Y}$.; $\mathrm{Li}, \mathrm{Z}$. Distributed optimal resource management based on the consensus algorithm in a microgrid. IEEE Trans. Ind. Electron. 2015, 62, 2584-2592. [CrossRef]

13. Hug, G.; Kar, S.; Wu, C. Consensus + innovations approach for distributed multiagent coordination in a microgrid. IEEE Trans. Smart Grid 2015, 6, 1893-1903. [CrossRef]

14. Han, R.; Meng, L.; Ferrari-Trecate, G.; Coelho, E.A.A.; Vasquez, J.C.; Guerrero, J.M. Containment and consensus-based distributed coordination control to achieve bounded voltage and precise reactive power sharing in islanded AC microgrids. IEEE Trans. Ind. Appl. 2017, 53, 5187-5199. [CrossRef]

15. Chen, G.; Zhao, Z. Delay effect on consensus-based distributed economic dispatch algorithm in microgrid. IEEE Trans. Power Syst. 2018, 33, 602-612. [CrossRef] 
16. Zhao, T.; Ding, Z. Distributed agent consensus-based optimal resource management for microgrid. IEEE Trans. Sustain. Energy 2018, 9, 443-452. [CrossRef]

17. Zhang, X.; Xu, H.; Yu, T.; Yang, B.; Xu, M. Robust collaborative consensus algorithm for decentralized economic dispatch with a practical communication network. Electr. Power Syst. Res. 2016, 140, 597-610. [CrossRef]

18. Liu, N.; Wang, J.; Wang, L. Distributed energy management for interconnected operation of combined heat and power-based microgrids with demand response. J. Mod. Power Syst. Clean Energy 2017, 5, 478-488. [CrossRef]

19. Zhang, G.; Cao, Y.; Cao, Y.; Li, D.; Wang, L. Optimal energy management for microgrids with combined heat and power (CHP) generation, energy storage, and renewable energy sources. Energies 2017, 10, 1288. [CrossRef]

20. Zhang, Z.; Chow, M.-Y. Convergence analysis of the incremental cost consensus algorithm under different communication network topologies in a smart grid. IEEE Trans. Power Syst. 2012, 27, 1761-1768. [CrossRef]

21. Gibbons, A. Algorithmic Graph Theor; Cambridge University Press: Cambridge, UK, 1985.

22. Walters, D.C.; Sheble, G.B. Genetic algorithm solution of economic dispatch with value point loading. IEEE Trans. Power Syst. 1993, 8, 1325-1332. [CrossRef]

23. Duvvuru, N.; Swarup, K.S. A hybrid interior point assisted differential evolution algorithm for economic dispatch. IEEE Trans. Power Syst. 2011, 26, 541-549. [CrossRef]

24. Erdeljan, A.; Capko, D.; Vukmirovic, S.; Bojanic, D.; Congradac, V. Distributed PSO algorithm for data model partitioning in power distribution systems. J. Appl. Res. Technol. 2014, 12, 947-957. [CrossRef]

(C) 2018 by the authors. Licensee MDPI, Basel, Switzerland. This article is an open access article distributed under the terms and conditions of the Creative Commons Attribution (CC BY) license (http:/ / creativecommons.org/licenses/by/4.0/). 